\title{
Cerebrovascular Lesions in Alzheimer-associated Diseases - A Neuropathological Study with 7.0-tesla Magnetic Resonance Imaging
}

\author{
Jacques De Reuck \\ Degenerative and Vascular Cognitive Disorders, Université de Lille 2, INSERM U1171, Lille, France
}

DOI: https://doi.org/10.17925/ENR.2018.13.2.100

$\mathrm{M}$ ixed dementias are clinically under-recognised and need neuropathological confirmation. The most frequent types are those composed of Alzheimer's dementia (AD) associated with cerebral amyloid angiopathy (CAA), cerebral arteriosclerotic micro-angiopathy (CAMA) and Lewy body disease (LBD). The present neuropathological study with 7.0-tesla magnetic resonance imaging (MRI) compares the impact and the distribution of cerebrovascular lesions between unmixed AD brains to those with different types of associated disorders. Twenty-six unmixed AD brains were compared to 12 associated with LBD, eight with CAMA and 24 with CAA. On neuropathological examination brains with AD-CAA had the most severe cerebrovascular lesions, those with AD-CAMA had a higher number of lacunes and in the AD-LBD brains only an increase of cortical micro-infarcts was observed. On MRI examination, white matter changes were only increased in the AD-CAA group. Cortical micro-infarcts were significantly more frequent in all the sections of the AD-CAA and AD-CAMA groups. In the AD-LBD brains, they were only moderately more common in the occipital section. Cortical micro-bleeds were observed in all mixed dementia cases. A moderate amount of superficial siderosis was only seen in the AD-CAA brains. The present study confirms our previous findings that CAA and CAMA are the main causes of occurrence of different cerebrovascular lesions in the mixed AD brains.

\section{Keywords}

Alzheimer's disease, Lewy body disease, cerebral arteriosclerotic micro-angiopathy, cerebral amyloid angiopathy, post-mortem cerebrovascular lesions, 7.0-tesla magnetic resonance imaging

Disclosure: Aside from being a member of the journal's editorial board, Jacques L De Reuck has nothing to declare in relation to this article.

Acknowledgements: The following persons have contributed to the present research project: Florent Auger, Nicolas Durieux, Vincent Deramecourt, Charlotte Cordonnier, Claude-Alain Maurage, Didier Leys, Florence Pasquier and Regis Bordet. This study was funded by the INSERM U 1172 (Université Lille 2, Lille, France).

Compliance with Ethics: All procedures were followed in accordance with the responsible committee on human experimentation and with the Helsinki Declaration of 1975 and subsequent revisions. A previously obtained informed consent of the patients, or from the nearest family, allowed an autopsy for diagnostic and scientific purposes. The brain tissue samples were acquired from the Lille Neuro-Bank of the Lille University, federated to the 'Centre des Resources Biologiques' that acted as an institutional review board. Authorship: The named author meets the International Committee of Medical Journal Editors (ICMJE) criteria for authorship of this manuscript, takes responsibility for the integrity of the work as a whole, and has given final approval to the version to be published.

Open Access: This article is published under the Creative Commons Attribution Noncommercial License, which permits any non-commercial use, distribution, adaptation and reproduction provided the original author(s) and source are given appropriate credit. (c) The Authors 2018.

Received: 9 October 2018

Accepted: 30 November 2018

Citation: European Neurological Review. 2018;13:(2):100-2

Corresponding Author: Jacques De Reuck,

Ryvisschepark 16, BE-9052 Zwijnaarde,

Belgium. E: dereuck.j@gmail.com

Support: No funding was received in

the publication of this article.
The occurrence of mixed dementias is significantly related to the aging process. ${ }^{1}$ Their brain pathology accounts for most cases in community-dwelling older persons. ${ }^{2}$ Mixed dementias are clinically under-recognised and need neuropathological confirmation. ${ }^{3}$ The most frequent types are those composed of Alzheimer's dementia (AD) associated with cerebral amyloid angiopathy (CAA), cerebral arteriosclerotic micro-angiopathy (CAMA), and Lewy body disease (LBD). ${ }^{4.5}$ AD-CAA is by far the most frequent association, ${ }^{6}$ while CAA mainly involves the leptomeninges and the cerebral cortex, and CAMA mainly affects the basal ganglia with white matter changes. ${ }^{7}$ Cortical micro-infarcts are not only frequent in AD-CAA but also in LBD., ${ }^{8,9}$ Additionally, cortical micro-bleeds are increased in the mixed dementia diseases. ${ }^{10}$

Post-mortem magnetic resonance imaging (MRI) is an additional tool of the neuropathological examination of neurodegenerative and cerebrovascular diseases, as it allows quantification and location of white matter changes, cortical micro-infarcts, cortical micro-bleeds and iron deposits, and is able to detect the areas of cortical superficial siderosis." The present neuropathological study with 7.0-tesla MRI compares the impact and the distribution of cerebrovascular lesions between unmixed $A D$ brains to those with the different types of associated disorders.

\section{Material and methods}

A total of 80 patients with unmixed and mixed AD, who had been followed up at the Lille University Hospital, underwent an autopsy. Twenty-six unmixed AD brains were compared to 12 associated with $L B D$, eight with CAMA and 24 with CAA. Mean age, gender distribution and the different types of cerebrovascular lesions were compared.

The standard diagnostic procedure consisted of examining samples from the primary motor cortex, the associated frontal, temporal and parietal cortex, the primary and secondary visual cortex, the cingulate gyrus, the basal nucleus of Meynert, the amygdaloid body, the hippocampus, basal ganglia, mesencephalon, pons, medulla and cerebellum. Slides from paraffin embedded sections were stained with haematoxylin-eosin, luxol fast blue and Perl. Immune-staining for protein tau, $\beta$-amyloid, $\alpha$-synuclein, prion protein, TDP-43 and ubiquitin was also performed.

AD features were classified according to the Braak and Braak criteria. ${ }^{12}$ The main diagnosis of $A D$ was retained when stages $V$ and $V I$ were reached. The criteria of a consensus protocol were used to assess the severity of CAA. ${ }^{13}$ The degree of CAA was evaluated semi-quantitatively on four cortical samples and graded from $0-3$. Only brains with grade 3 in all samples were considered to have CAA. LBD was diagnosed according to the report of the consortium on DLB international 
Table 1: Comparison of demographic features and cerebrovascular lesions in post-mortem brains of patients with unmixed and mixed Alzheimer's disease

\begin{tabular}{|l|l|l|l|l|}
\hline & $\begin{array}{l}\text { AD } \\
n=26\end{array}$ & $\begin{array}{l}\text { AD-LBD } \\
n=12\end{array}$ & $\begin{array}{l}\text { AD-CAMA } \\
n=8\end{array}$ & $\begin{array}{l}\text { AD-CAA } \\
n=24\end{array}$ \\
\hline $\begin{array}{l}\text { Patient characteristics } \\
\text { Age, year } \\
\text { (Standard deviation) }\end{array}$ & $76(10)$ & $75(13)$ & $79(8)$ & $79(9)$ \\
\hline $\begin{array}{l}\text { Male gender, \% } \\
\text { Neuropathology }\end{array}$ & 47 & 40 & 63 & 33 \\
\hline White matter changes & $0.9(0.9)$ & $0.4(0.9)$ & $0.9(0.6)$ & $1.7\left(1.0^{\dagger}\right.$ \\
\hline $\begin{array}{l}\text { Lacunar infarct } \\
\text { Territorial infarct }\end{array}$ & $0.2(0.6)$ & $0.0(0.0)$ & $0.9(0.3)^{\star}$ & $0.3(0.7)$ \\
\hline Lobar haematoma & $0.1(0.3)$ & $0.2(0.4)$ & $0.5(0.8)$ & $0.3(0.7)$ \\
\hline Cortical micro-infarct & $0.1(0.4)$ & $1.4(0.5)^{\dagger}$ & $1.1(0.8)^{\dagger}$ & $2.9(0.8)^{\ddagger}$ \\
\hline Cortical micro-bleed & $0.7(0.6)$ & $1.2(0.8)$ & $1.8(0.7)^{\star}$ & $2.8(0.8)^{\ddagger}$ \\
\hline
\end{tabular}

$* p<0.05$ and $>0.01 \cdot+p \leq 0.01 * \neq p<0.001$

$A D=$ Alzheimer's disease; $C A A=$ cerebral amyloid angiopathy; $C A M A=$ cerebral arteriosclerotic micro-angiopathy; $L B D=$ Lewy body disease.

workshop. ${ }^{14}$ Staging of the CAMA pathology was performed according to the recommendations of the vascular dementia group. ${ }^{15}$

\section{Neuropathological examination}

In addition to the detection of macroscopic visible lesions such as haematomas and territorial and lacunar infarcts, a whole coronal section of a cerebral hemisphere, at the level of the mamillary body, was taken for semi-quantitative microscopic evaluation of the small cerebrovascular lesions such as white matter changes, cortical micro-bleeds, cortical micro-infarcts, and lacunes. The mean values of white matter changes were the average of the ranking scores: no change (R0), a few isolated (R1), frequently scattered in the corona radiata (R2), and forming confluent lesions (R3) of myelin and axonal loss. For the other cerebrovascular lesions, their mean values corresponded to their average numbers in the individual brains.

\section{Magnetic resonance image examination}

A 7.0-tesla MRI (Bruker BioSpin SA, Ettlingen, Germany) was used with an issuer-receiver cylinder coil of $72 \mathrm{~mm}$ inner diameter (Ettlingen, Germany), according to a previously described method. ${ }^{16}$ Three coronal sections of a cerebral hemisphere were submitted to SPIN ECHO T2 and T2* MRI sequences: a frontal, a central and a parieto-occipital one. The ranking scores of severity of white matter changes were evaluated separately on different brain sections using the same method as was performed on the neuropathological section. The number of the other small cerebrovascular lesions was also determined by consensus evaluation. The incidence of cortical superficial siderosis, not associated to a visible underlying lesion, was also evaluated on the $T 2^{*}$ sequence. ${ }^{17}$

\section{Statistical analyses}

Univariate comparisons of unpaired groups were performed with the Fisher's exact test for categorical data. The non-parametric Mann-Whitney U-test was used to compare continuous variables. The significance level, two-tailed, was set at $\leq 0.05$ for moderately significant, at $\leq 0.01$ for significant and at $\leq 0.001$ for highly significant.

\section{Results}

Age and gender distribution were not statistically different between the unmixed and mixed dementia groups. On neuropathological examination,
Figure 1: SPIN-ECHO T2 and T2* imaging from an occipital coronal section of a brain with mixed Alzheimer's and Lewy body diseases

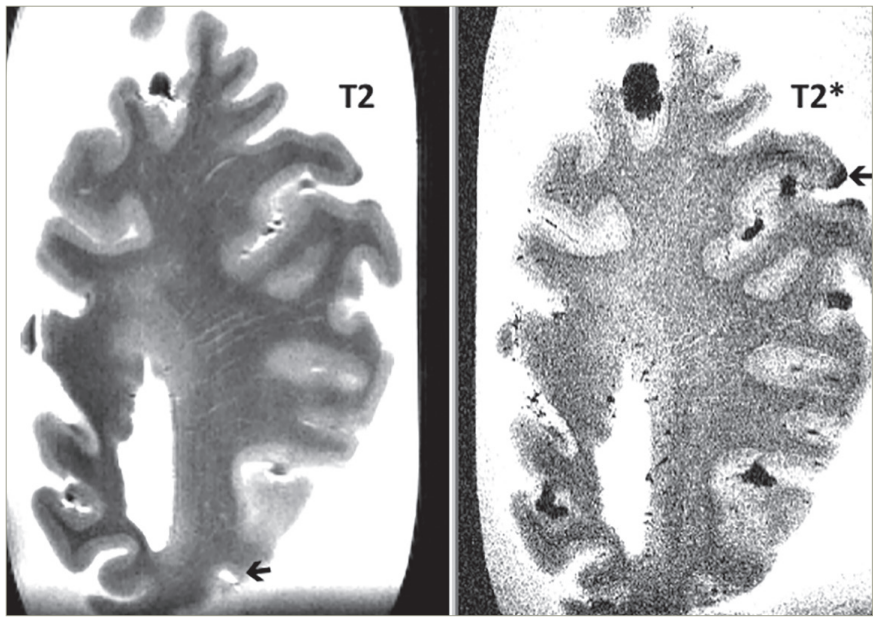

Note the diffuse periventricular hyperintensities of the white matter and a cortical micro-infarct (arrow) on the T2 sequence. A small cortical micro-bleed is seen on the T2* sequence (arrow).

Figure 2: SPIN-ECHO T2 and T2* imaging from a frontal coronal section of a brain with mixed Alzheimer's disease and cerebral arteriosclerotic micro-angiopathy

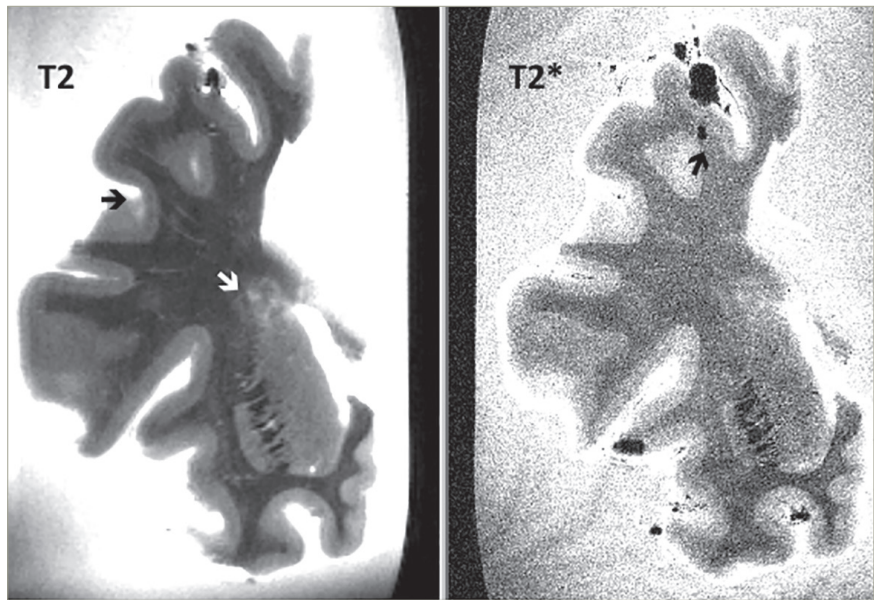

Some periventricular lacunar infarcts (white arrow) and a cortical micro-infarct (black arrow) are present on the T2 sequence. A cortical micro-bleed (black arrow) is observed on $\mathrm{T}_{2} *$ sequence.

patients with AD-CAA had the most severe white matter changes and the highest incidence of lobar haematomas, cortical micro-infarcts and cortical micro-bleeds compared to the unmixed group. Patients with AD-CAMA had a significantly higher number of lacunes and an increase in cortical micro-infarcts and cortical micro-bleeds. In the AD-LBD brains only an increase of cortical micro-infarcts was observed compared to the unmixed group (Table 1).

On MRI examination, white matter changes were only increased in the AD-CAA group, mainly in the central section. Cortical micro-infarcts were significantly more present in all the sections of the AD-CAA and AD-CAMA groups. In the AD-LBD, brains they were only moderately more common in the occipital section, compared to the unmixed $A D$ group (Figure 1). Cortical micro-bleeds were significantly more frequent in all the sections of AD-CAA and AD-CAMA brains (Figure 2), while in the AD-LBD group they occurred more frequently in the frontal section and, to a lesser degree, in the central one. Although cortical superficial 
Figure 3: SPIN-ECHO T2 and T2* imaging from a frontal coronal section of a brain with mixed Alzheimer's disease and cerebral amyloid angiopathy
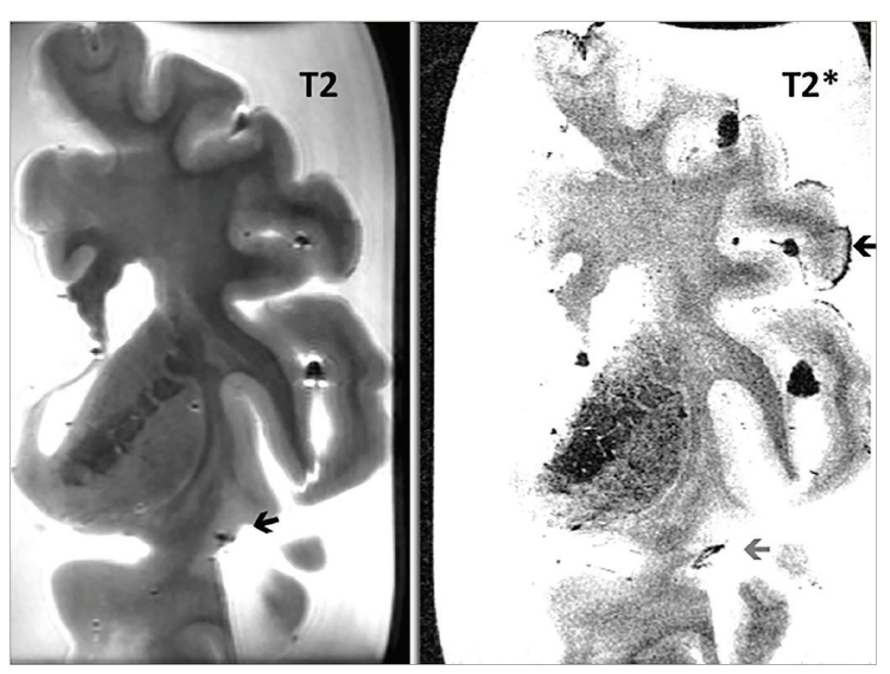

On T2 and T2* sequences a cortical micro-bleed is present (grey arrow). A moderate area of cortical superficial siderosis (black arrow) is observed on the T2* sequence.

siderosis was only observed in the AD-CAA brains (Figure 3) but not in the other mixed and unmixed AD diseases, the incidence was too low to be considered as statistically significant (Table 2).

\section{Discussion}

The present study confirms our earlier findings that CAA and CAMA are the main causes of occurrence of different cerebrovascular lesions in mixed $A D$ brains. ${ }^{18,19}$ However, now we have also differentiated the impact of these lesions between AD-CAA and AD-CAMA, with a more severe cerebrovascular burden in the former group. In addition, it is shown that lacunar infarcts are more frequent in the AD-CAMA group, as already demonstrated in pure vascular dementia brains. ${ }^{20}$ Lacunar infarcts are mainly observed in the centrum semiovale. ${ }^{21}$

Lewy body pathology is associated with $A D$ in $25 \%$ of cases. $^{22}$ As previously shown in the unmixed LBD, an increase of cortical micro-infarcts and cortical micro-bleeds in the mixed AD-LBD group is observed. ${ }^{19}$ The cortical micro-infarcts are more frequent in the occipital section and the cortical micro-bleeds mainly in the frontal section. These
Table 2: Comparison of the frequency and the distribution of white matter changes, cortical micro-infarcts, cortical micro-bleeds and cortical superficial siderosis between unmixed and mixed Alzheimer's disease

\begin{tabular}{|c|c|c|c|c|}
\hline & $\begin{array}{l}A D \\
n=26\end{array}$ & $\begin{array}{l}\text { AD-LBD } \\
n=12\end{array}$ & $\begin{array}{l}\text { AD-CAMA } \\
n=8\end{array}$ & $\begin{array}{l}\text { AD-CAA } \\
n=24\end{array}$ \\
\hline \multicolumn{5}{|c|}{ White matter changes } \\
\hline Frontal & $0.7(0.7)$ & $0.5(0.7)$ & $1.0(0.6)$ & $1.2(0.7)^{*}$ \\
\hline Central & $0.6(0.8)$ & $0.8(0.9)$ & $0.3(0.7)$ & $1.6(0.8)^{\ddagger}$ \\
\hline Occipital & $0.8(0.9)$ & $1.3(0.7)^{*}$ & $0.3(0.5)$ & $1.7(1.0)^{\star}$ \\
\hline \multicolumn{5}{|c|}{ Cortical micro-infarcts } \\
\hline Frontal & $0.3(0.5)$ & $0.7(0.7)$ & $2.7(0.6)^{\ddagger}$ & $1.8(0.9)^{\ddagger}$ \\
\hline Central & $0.3(0.5)$ & $0.6(0.8)$ & $1.7(0.5)^{\ddagger}$ & $2.0(0.8)^{\ddagger}$ \\
\hline Occipital & $0.6(0.7)$ & $1.3(0.9)^{*}$ & $3.7(0.4)^{\ddagger}$ & $2.4(0.9)^{\ddagger}$ \\
\hline \multicolumn{5}{|c|}{ Cortical micro-bleeds } \\
\hline Frontal & $0.8(0.8)$ & $1.9(0.8)^{\dagger}$ & $1.8(0.6)^{\dagger}$ & $2.5(0.7)^{\ddagger}$ \\
\hline Central & $0.9(0.9)$ & $1.7(0.9)^{*}$ & $2.7(0.4)^{\ddagger}$ & $3.0(0.8)^{\ddagger}$ \\
\hline Occipital & $0.9(0.9)$ & $1.3(0.9)$ & $2.7(0.6)^{\ddagger}$ & $2.8(0.7)^{\ddagger}$ \\
\hline \multicolumn{5}{|c|}{ Cortical superficial siderosis } \\
\hline Frontal & $0.0(0.0)$ & $0.0(0.0)$ & $0.0(0.0)$ & $0.3(0.6)$ \\
\hline Central & $0.0(0.0)$ & $0.0(0.0)$ & $0.0(0.0)$ & $0.3(0.6)$ \\
\hline Occipital & $0.0(0.0)$ & $0.0(0.0)$ & $0.0(0.0)$ & $0.3(0.4)$ \\
\hline
\end{tabular}

Images obtained using nuclear magnetic resonance imaging.

values in brackets: standard deviation.

${ }^{*} p \leq 0.05$ and $>0.01 ;{ }^{*} p \leq 0.01 ; \neq p \leq 0.001$

$A D=$ Alzheimer's disease; $C A A=$ cerebral amyloid angiopathy; $C A M A=$ cerebral arteriosclerotic micro-angiopathy; $L B D=$ Lewy body disease.

results are different from those observed in a clinical study with 3.0-tesla $\mathrm{MRI}$, in which cortical micro-bleeds are more frequently observed in the occipital lobes. ${ }^{23}$ The impact of associated CAA is limited to a further increase of cortical micro-infarcts in the frontal sections. ${ }^{24}$ The moderate amount of superficial siderosis, observed in the AD-CAA brains, is related to underlying cortical haemorrhagic or ischaemic lesions. ${ }^{25}$ Superficial siderosis is now included in the Boston criteria for CAA. ${ }^{26}$ AD-CAA brains have less severe cerebrovascular lesions than in unmixed CAA, probably due to some protective effect of the neurodegenerative disease. ${ }^{27}$ Ultimately, the clinical influence of co-occurring pathologies on disease progression mainly depends on the severity of the AD pathology.
De Reuck J, Maurage C-A, Deramecourt V, et al. Aging and cerebrovascular lesions in pure and in mixed neurodegenerative and vascular dementia brains: a neuropathological study. Folia

Neuropathol. 2018;56:81-7. pathologies account for most dementia cases in comm
dwelling older persons. Neurology. 2007:69:2197-204.

dwelling older persons. Neurology. 2007:69:2197-204.
Zekry D, Duyckaert C, Belmin J, et al. Alzheimer's disease and brain infarcts in the elderly. Agreement with neuropathology.
I Neurol 2002:249:1529-34.

Magaki S, Yong WH, Khanlou N, et al. Comorbidity in dementia: update of an ongoing autopsy study. J Am Geriatr SOC.

Pillai JA, Butler RS, Bonner-Jackson A, Leverenz JB. Impact of (t) Cementia in a national autopsy

De Reuck J, Deramecourt V, Cordonnier C, et al. The impact of cerebral amyloid angiopathy on the occurrence of featurs: a neuroparith Thal DR Ghebremedhin E, Orantes M Wiestler OD Vascur pathology in Alzheimer's disease: correlation of cerebral amy oid

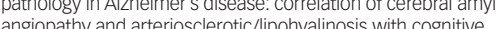
angiopathy and arteriosclerotichiponyalinosis with cognitive

Soontomniyomkii $V$ Lynch MD Mermesh S et a Cereb Soontomniyomis, Lynch MD, Mermash s, et al. Cerebral angiopathy. Brain Pathol. 2010;20:459-67.

7.0-tesla magnetic resonance study of cortical microinfarcts in neurodegenerative diseases and vascular dementia with in neurodegeneicithe diseases and vascular dementia with neuropathological correlates. J Neurol SCI. 2014;346:85-9. cerebrovascular lesions in patients with Lewy body dementia: a neuropathological study. Clin Neurol Neurosurg. 2013;115: 1094-7.

11. De Reuck J. Post-mortem magnetic resonance imaging as an additional tool of the neuropathological examination of neurodegenerative and cerebrovascular diseases. Eur Neuro Rev. 2016,11.22-5.

Brak $\mathrm{H}$, Braak E. Neuropathological staging of Azhemer-related Changes. Acta Neuropathol. 1991;82:239-59. vove S, Chalmers K, ince, et al. Development, appraisal, validation and implementation of a consensus protocol for are brain issue. Am JNeurodegener Dis. 2014,310-32.

suidelines for the clinical and pathologic diagnosis of dementia with Lewy bodies (DLB): report of the consortium on DLB international workshop. Neurology. 1996;47:1113-24.

15. MCAleese KE, Alafuzoff I Charidimou A, et al Post-mortem

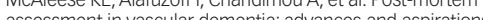
assessment in vascular

16. De Reuck JAuger F, Cordonnier $C$ et al Comparison of $7.0-T T^{\star}$ magnetic brain sections of Alzheimer patients with their neuropathological

( siderosis of the central nervous system: a post-mortem 7.0-tesla magnetic resonance imaging study with neuropathological

corelates. Cerebrovasc Dis. 2013,36:412-7.

De Reuck J, Deramecourt V, Cordonnier C, et al. The incidence of post-mortem neurodegenerative and cerebrovascular pathology

in mixed dementia. J Neurol Sci. 2016;366:164-6.

De Reuck J, Deramecourt V, Auger F, et al. Cerebrovascular lesions in mixed neurodegenerative dementia: a . 2017;78:1-5.
20. De Reuck J, Auger F, Durieux N, et al. Frequency and topography of small cerebrovascular lesions in vascular and mixed dementia: a post-mortem 7-tesla magnetic resonance imagin study with neu

21. De Reuck J, Auger F, Durieux N, et al. Topographic distribution of white matter changes and lacunar infarcts in neurodegenerative white matter changes and lacunar infarcts in neurodegenerative and vascular dementia syndromes. A post-mortem 7.0-tesla

22. Bergeron C, Pollanen M. Lewy bodies in Alzheimer disease - one Bergeron C, Pollanen M. Lewy bodies in Alzheimer disease - one
or two diseases? Alzheimer Dis Assoc Disord. 1989;3:197-204.

3. Gungor I, Sarro L, Graff-Radford J, et al. Frequency and topography of cerebral microbleeds in dementia with Lewy bodies compared to Alzheimer's disease. Parkinsonism Relat Disord. 2015;21:1101-4.

24. De Reuck J Auger $F$ Durieux $N$, et al The impact of cerebral amyloid angiopathy in Lewy body dementia: a neuropathological study with magnetic resonance imaging correlations. Journal of Neurodegenerative Disorders \& Research. 2018;1:101.

5. De Reuck J, Deramecourt V, Cordonnier C, et al. Superficial siderosis of the central nervous system: a post-mortem 7.0-tesla magnetic resonance imaging study with neuropathological

26. Charidimou A, Linn J, Vernooy MW et al. Cortical superficial siderosis: detection and clinical significance in cerebrat sidersis. detection and realigitions 2015;138:2126-39.

27. De Reuck J, Cordonnier C, Auger F, et al. Cerebrovasular lesions in cerebral amyloid angiopathy with and without Alzheimer's disease: a neuropathological study with post-mortem 7.0 -tes disease. a neuropath logical study wh post-mortem 7.0-tes magnetic resonance imaging. EC Neurology. 2018;954-60.

28. Brenowitz WD, Hubbard RA, Keene CD, et al. Mixed in a large autopsy sample. Alzheimers Dement. 2017;13:654-62. 